\title{
A new perspective in the road asset management with the use of advanced monitoring system \& BIM
}

\author{
Salvatore Cafiso ${ }^{1}$, Alessandro Di Graziano ${ }^{1}$, Carmelo D’Agostino ${ }^{1,}$, Giuseppina \\ Pappalardo $^{1}$, and Emanuele Delfino ${ }^{1}$ \\ ${ }^{1}$ University of Catania, Dept. of Civil Eng. \& Arch., via S. Sofia, 6495125 - Catania (CT), Italy
}

\begin{abstract}
The present paper reports different applications which have a common data source: Automatic Road Analyzer (ARAN) of the Transport Infrastructure Laboratory of the University of Catania. Data surveyed with ARAN were used to develop performance indicators of the road asset. A BIM model makes possible to improve the flexibility in information access and to merge data with the aim to allow a complete Road Asset Management (RAM) application in the same environment Data Collected and stored with high efficiency equipment allows to get high quality and detailed information of the road asset and offers the chance to create georeferenced database which can be used both for safety assessment and for the other RAM related applications. BIM provides a comprehensive approach involving the generation and management of transport infrastructure assets using 3D digital representations of their physical and functional characteristics allowing to produce ad hoc output for investigate in the same environment different parts of the RAM.
\end{abstract}

\section{Introduction}

Road network maintenance and safety are part of the Road Asset Management (RAM), but classically treated separately considering, other than different approaches, even different data or in general a different dataset organization. The typical approach to monitoring activities collects data for a specific purpose missing the opportunities for merging information and optimize the management process. In this framework, the introduction of new high efficiency equipment, based on advanced technology, for infrastructure survey and inventory and the contemporary development of BIM applications is changing the perspective in the management of road infrastructure [1].

The present paper reports different applications which have a common data source: Automatic Road Analyzer (ARAN) of the Transport Infrastructure Laboratory of the University of Catania. Data surveyed with ARAN were used develop performance indicators of the road asset and a BIM model makes possible to improve the flexibility in information access and to merge data with the aim to allow a complete RAM application in the same

\footnotetext{
* Corresponding author: carmelo.dagostino@unict.it
} 
environment Data Collected and stored with high efficiency equipment allows to get high quality and detailed information of the road asset and offers the chance to create georeferenced database which can be used both for safety assessment and for the other RAM related applications. BIM provides a comprehensive approach involving the generation and management of transport infrastructure assets using 3D digital representations of their physical and functional characteristics allowing to produce ad hoc output for investigate in the same environment different parts of the RAM.

\section{Equipment and data collection}

The Automatic Road Analyzer (ARAN) is one of the most efficient equipment that allow to collect data from pavement and infrastructure features with a productivity of $200 \mathrm{~km} /$ day. It allows to collect more than fifteen different data streams continuously and at varying capture rates in a single pass at traffic speed.

ARAN 9000 has recently been acquired by the University of Catania, which is carrying out an extensive research programme for system calibration and output adaptation to a performance-based inventory of road infrastructures and for the computation of performance indexes for pavement or infrastructure condition evaluation.

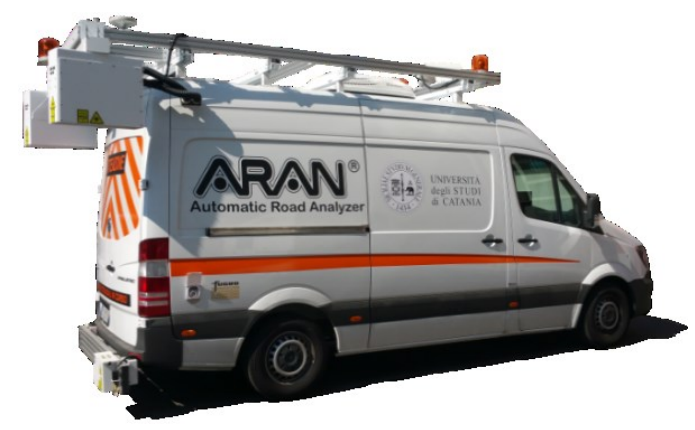

Fig. 1. ARAN 9000 of University of Catania.

The ARAN available at the transport Infrastructure laboratory of Catania University uses a Mercedes Sprinter vehicle where three PCs working in synchrony to collect data coming from the installed systems:

- SOP Laser, consisting of 2 laser to $16 \mathrm{kHz}$ LMI SLS5000-200/300 and 2 accelerometers PCB 3711.

- SmarlTexture measurement system, consisting of 1 laser $64 \mathrm{kHz}$ LMI Optocator 2008-128 to measure the macro texture of the road;

- LCMS measurement system, uses high speed cameras, featuring dedicated optics and 48 laser line projector to acquire either in 20 images (black and white images) or high resolution 30 profiles, giving three dimensions surface of pavement with millimeter accuracy;

- 2 GPS L2 (DGPS), OM/, /MU, 3 cameras (2 front and 1 rear).

It is fully integrated into a high roof chassis, it means that it is an advanced data collection vehicle equipped with a precision survey system and software able to perform the collection of road's geometric data (cross section, gradients, horizontal and vertical alignment), pavement unevenness, detection and classification of distress and road asset inventory data. It also takes care of the acquisition and filing of the images of accessory elements (for signals, advertising, rights of way, etc.). 
All the data recorded are accurately located using any geographical reference system (e.g. Gauss-Boaga or UTM) with the help of a satellite differential correction receiver (DGPS), coupled with an inertial platform, and they are associated with chainages coming from high resolution odometer (DMI), as well.

\section{ARAN application for RAM}

In this section, different possible survey applications which can be carried out with ARAN are reported shown that always a post elaboration of data is needed to reach a certain level of accuracy. ARAN' target, in commercial activities is mainly related to the pavement distress analysis and asset inventory production. However, using data from the acquisition and elaboration software the use of data from ARAN is limited to the format and typology of the close software output. Data from ARAN are of a high quality in terms of resolution and variety and they can be used as base data for RAM applications (Figure 2) considering always some effort of scientific research needed for adapting them to the scope.

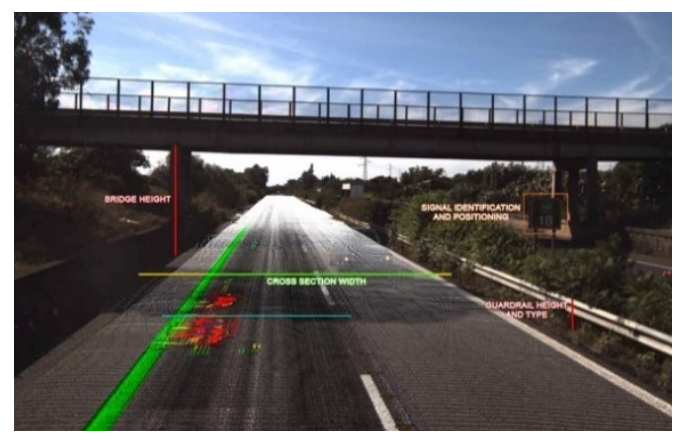

Fig. 2. Data can be surveyed with ARAN from different sensors installed on board.

\subsection{Asset inventory}

Asset inventory detection is carried out using the ARAN surveying vehicle in combination with the HD Videolog system and the Surveyor ${ }^{\circledR}$ asset extraction software. The high quality of the two front cameras and one rear camera with the resolution of 2750x2200 pixels ensure a complete detection of the road environment in a $240^{\circ}$ field of view (Figure 3 ). The Videolog and GPS systems work in sync to provide the geo-referencing of digital images. Moreover, the inertial aided navigation system ensures that GPS accuracy is maintained even during long satellite blackouts with sub-meter precision.
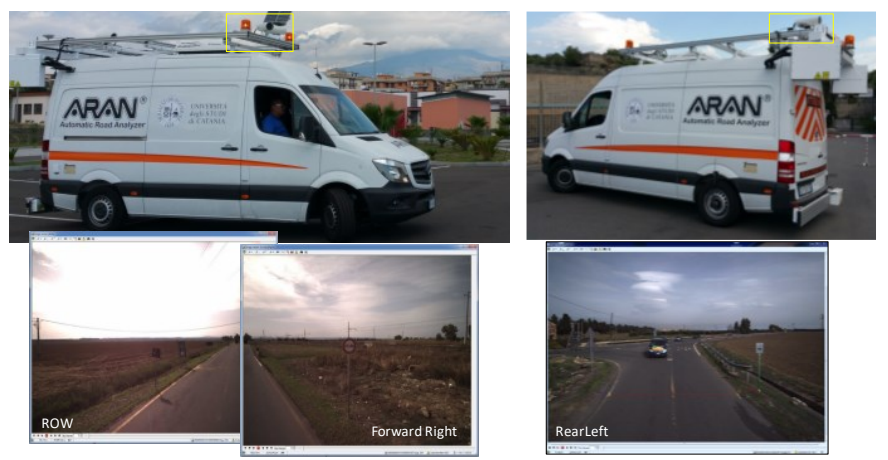

Fig. 3. Road Video Survey 
The analysis of images for extracting road inventory assets is carried out by using a software tool that allows to display, measure and inventory any road features located along the road and displayed in the image (e.g. lane, shoulder width, sign, marking, roadside, safety barriers, etc.) (Figure 4). More specifically, after the calibration of the camera system, it is possible to locate and measure the main dimensions (length, width) of any object along the roadway. Figure 4 presents an example of carriageway measurement and the collection of additional information about pavement and barriers [2].

A comprehensive asset inventory was defined able to include not only the base information required in relation of the national guidelines, but more data are needed for checking the compliance with design standards (e.g. geometric design, safety barriers, signs and markings) and, it can provide the base, to estimate the safety performance of the network $[3,4]$.

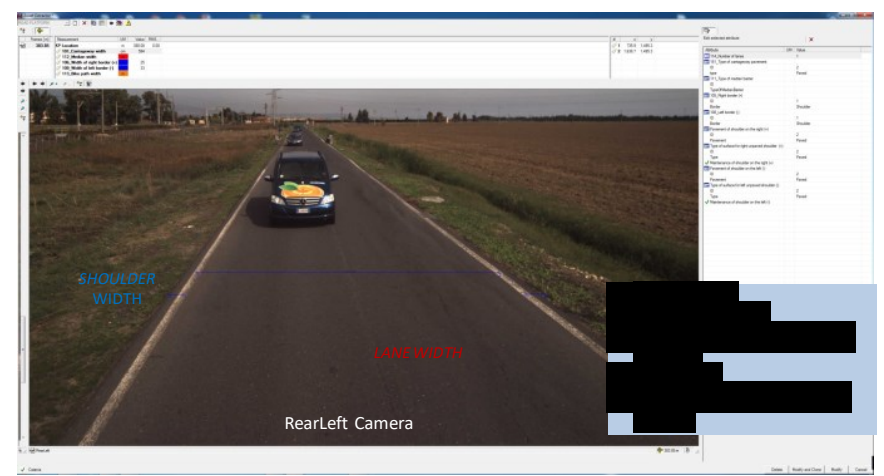

Fig. 4. Example of carriageway measurement

\subsection{Safety analysis (inspections)}

One of ARAN application in road safety field is Safety Inspection (SI), which is mandatory for European Union countries based on EU Directive [5]. In the framework of the IASP (Identificazione e Adeguamento delle Strade Pericolose) [6-8] research program co-financed by the European Commission in 2004 which provided guidelines for inspection [8] on local rural road, each single element part of an inspection was translated in number based on a score relate to its safety performance. In this way SIs can be used as a source of information capable of prioritising measures and the treatment of road sections in the prevention of traffic accidents. The last implementation of IASP project was to conduct a Road Safety Inspection based on road's geometric data (cross section, gradients, horizontal and vertical alignment) [9] by using a high-efficiency system, ARAN. The IASP procedure was adapted to employ the data collected by the system. The comparison between traditional method and the use of ARAN in conducting SI was carried out based on IASP procedure and reported in this section to show how the ARAN output data can be used for application useful to be included in a BIM model [9].

Specifically, images recorded by ARAN were analyzed with the Surveyor ${ }^{\circledR}$ software in order to obtain all the measures for assigning a score to the safety issues used in the IASP procedure. By applying algorithms, these measures have been transformed in the same three categories of judgment of the IASP score criteria.

Results reported in Figure 5 show that the highest differences were identified for the scores related to embankment, terminals and roadside obstacles. For lane and shoulder width when a different score was assigned, it was rarely (i.e. less than $10 \%$ of cases) that it equalled 2 (i.e. no problem vs. high score problem). 


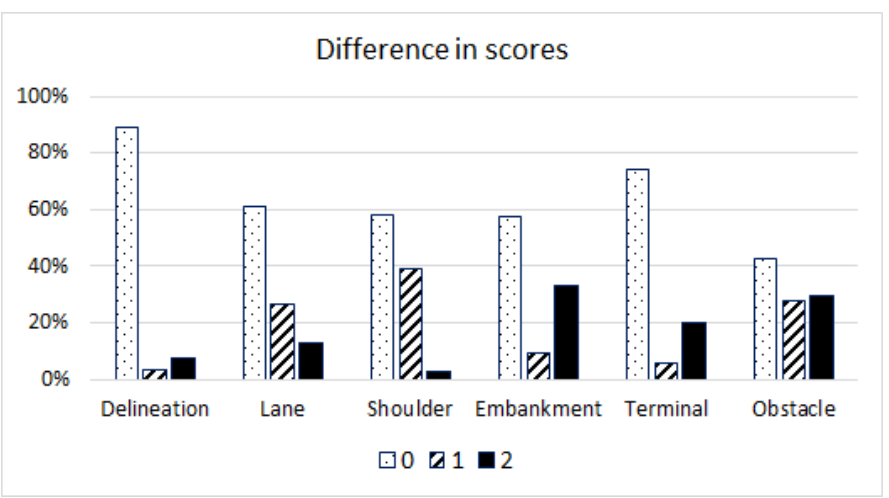

Fig. 5. Differences in score between the two procedures (absolute value of Score ARAN -Inspection).

The level of agreement in the results carried out by the two different methods has been estimated by a statistical test.

Specifically, the kappa statistic has been used [10]. The kappa coefficient (Cohen's kappa k) provides a measure of inter-rater agreement for categorical scales when there are two raters. If there is total agreement, $\mathrm{k}$ equals 1 . If there is no agreement other than that would be expected by chance, $\mathrm{k}$ equals 0 . A negative kappa value indicates disagreement between procedures. Moreover, it is possible to test whether the level of agreement is statistically significant. A level of significance of $10 \%(p$-value $=0.10)$ was assumed as acceptable for assuming a significant non-zero correlations at 90\%. Results are reported in Table 1.

Table 1. $\mathrm{k}$ statistics and $\mathrm{p}$-value of agreement between the two procedure.

\begin{tabular}{lcc}
\hline Safety issues & $\mathrm{k}$ & Significance \\
\hline Roadside: embankments & -0.073 & 0.032 \\
Roadside: dangerous terminals and & - & Insignificant data \\
transition & & \\
Roadside: obstacles & 0.118 & 0.012 \\
Cross section: lane width & -0.043 & 0.376 \\
Cross section: shoulder width & 0.175 & 0.000 \\
Delineation: chevrons & 0.108 & 0.022 \\
\hline
\end{tabular}

For shoulder width, the $\mathrm{k}$-value $(\mathrm{k}=0.175)$ and the statistical significance pointed out the highest correlation and agreement between the two procedures. Statistical agreement ( $p$ value $<0.1$ ) exists also for Delineation and roadside hazards related to Embankments and Obstacles, but $\mathrm{k}<0$ showed no rank correlation for Embankment, as well. For dangerous terminals/transition, collected data resulted not significant for performing the test because the judgment expressed by both groups assumed an almost constant value along the test roads.

\subsection{Pavement analysis}

The development of Laser Crack Measurement Systems (LCMS) introduced the advantage to get information only from the pavement surface with a higher point density and detail at higher survey speed. The technology of LCMS allows to survey several transversal sections of the pavement surface in the direction of motion [11]. This shape data is available every $5 \mathrm{~mm}$ along the road in the direction of travel, at $1 \mathrm{~mm}$ transverse resolution. The LCMS system employs high speed cameras, custom optics, and laser line projectors to acquire 2D images and high resolution 3D profiles of road surfaces that allow for automatic detection of 
cracks and the evaluation of macrotexture and other road surface features. Designed for both day and nighttime operation in all types of lighting conditions, the system is immune to sun and shadows and is capable of measuring pavement types ranging from concrete to dark asphalt. The LCMS can be operated at speeds of up to $100 \mathrm{~km} / \mathrm{h}$ on road lanes as wide as 4 $\mathrm{m}$. Each transversal profile, given by a series of points generated by LCMS is univocally identified by the linear chainage and GPS global position. Collected data can be processed with analysis software to automate the characterization a wide range of distresses (mainly, cracks, ruts, potholes, raveling, macro-texture) [12,13]. Longitudinal unevenness is collected by more traditional class 1 laser profilers with accuracy complying the ASTM standards [14].

The available software used e.g. by FUGRO ${ }^{\circledR}$ and Pavemetrics ${ }^{\circledR}$ for processing the LCMS provide an automatic detection of distresses on the pavement surface by combining the use of the images and of the 3D analysis of the points generated by LCMS (Figure 6).

The 3D texture of the pavement surface, generated by LCMS, contains enough information for detecting, measure and classify different distress basing on a flexible setting of several parameters that need operator expertise and calibration trials, as well. Looking e.g. to the PCI classification, LCMS can be used for identification and rating of a large amount of the 17 distresses included in the model such as joint reflection cracking, Lane/shoulder Drop off, potholes, rutting, raveling and all the surface and edge cracking. While few distresses need to be classified and rated manually, such as bleeding, patching, polished aggregate. Another set of pavement surface characteristics of PCI is related to unevenness. Those distresses are: bumps and sags, corrugation, railroad crossing, shoving, swell and depression. As mentioned, those irregularities in the pavement surface are not univocally correlated to the IRI automatically calculated from the laser profiles.

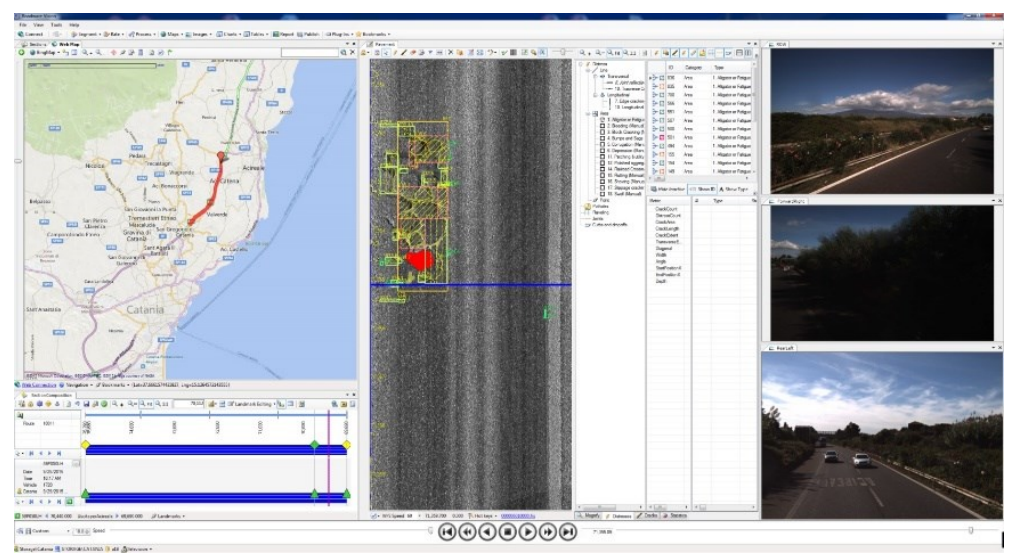

Fig. 6. Vision software ${ }^{\circledR}$

Different indexes were evaluated using data from ARAN by the team of the University of Catania. Mainly the research focused on the PCI, elaborating a procedure for the identification and rating of those distresses related to IRI but cannot be detected automatically by the software. Another application was related to the identification and rating of the Italian distress Catalogue [15]. With the aim to validate the proposed semi-automated methodology for the detection, classification and rating of distresses which cannot be detected automatically by the system a comparison with the manual detection was performed.

After several trials with failing of traditional statistical regression approach, only a nonparametric classifier (the Probabilistic Neural Network, PNN) was able to provide acceptable results pointing out the complexity of the problem and encouraging to further investigate the topic [16]. 
However, the system and analysis methodology is flexible enough to be adapted, after opportune analysis and validation, to different distresses catalogue and indexes with distress classification and rating based on real characteristic measures or ride quality.

\section{The advantage of using data from ARAN in a BIM model}

Building information modeling (BIM) is a process involving the generation and management of digital representations of physical and functional characteristics of places to support decision-making regarding any asset. BIM deals with the issue of information interoperability for preventing information loss in every phase of the lifecycle of the asset

Many projects are still being executed the traditional way. This has major disadvantages regarding information exchange in comparison to a collaborative BIM based process. Figure 7 shows that information gets lost in every phase of the project

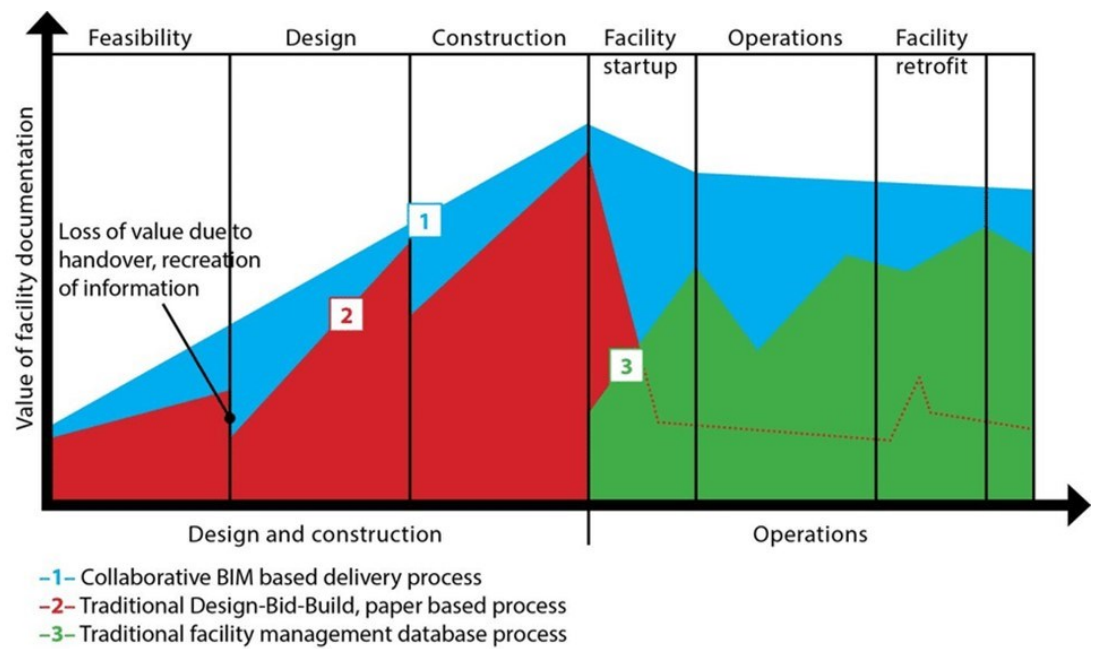

Fig. 7. Information Exchange during the lifetime of any asset [17].

ARAN data treatment and processing was modelled in a collaborative BIM based delivery process with the aim to fill the gap of any road where BIM was not available from the construction phase using high quality data collection. Moreover, the management of toad network in operation with ARAN permits to track the entire BIM asset life cycle.

\section{Conclusions}

The high accuracy of the measurements, the less time needed for the survey, the limited cost of the survey and high variety and quality of data collected by ARAN provide a flexible and high-quality dataset of information opening new perspective for the development of performance indicators. At the same time BIM delivers benefits throughout the entire asset life cycle. A BIM model can contain information/data on design, construction, logistics, operation, maintenance, budgets, schedules and much more than traditional databases or GIS.

\section{References}

1. Building Information Modelling (BIM) for Transport and Main Roads A guide to enabling BIM on Road Infrastructure Projects. State of Queensland (Department of Transport and Main Roads, 2017) 
2. S. Cafiso, C. D'Agostino, B. Persaud, Investigating the influence on safety of retrofitting Italian motorways with barriers meeting a new EU standard, Traffic Inj. Prev. 18 pp. 324-329 (2017). doi:10.1080/15389588.2016.1203424

3. S. Cafiso, C. D'Agostino, M. Kiec, G. Pappalardo, Surrogate measure of safety from road inspection data: experimental test on polish roads, Roads Bridg. - Drog. i Most. 16 pp. 115-130 (2017)

4. S. Cafiso, A. Di Graziano, Surrogate Safety Measures for Optimizing Investments in Local Rural Road Networks, Transp. Res. Rec. J. Transp. Res. Board. 2237 pp. 20-30 (2011). doi:10.3141/2237-03

5. Directive 2008/96/EC of the European Parliament and of the Council of 19 November 2008 on road infrastructure safety management, http://eurlex.europa.eu/LexUriServ/LexUriServ.do?uri=CELEX:32008L0096:EN:NOT (2008)

6. S. Cafiso, G. la Cava, A. Montella, G. Pappalardo, A Procedure to Improve Safety Inspections Effectiveness and Reliability on Rural Two-Lane Highways, Balt. J. Road Bridg. Eng. 1(3) pp. 143-150 (2006)

7. S. Cafiso, A. Di Graziano, G. Pappalardo, Safety Inspection and Management Tools for Low-Volume Road Network, Transp. Res. Rec. J. Transp. Res. Board. 2472 pp. 134141 (2015). doi:10.3141/2472-16

8. S. Cafiso, M. Kieć, M. Milazzo, G. Pappalardo, F. Trovato, Application of Safety Inspections for Evaluation of Two-Lane Regional Roads in Poland, Arch. Civ. Eng. 60 pp. 453-473 (2014). doi:10.2478/ace-2014-0031

9. S. Cafiso, M. Kiec, G. Pappalardo, Innovative methods for improving the effectiveness of road safety inspection, in: VI Int. Symp. Transp. Commun. NEW HORIZONS, (Doboj, Serbia, 2017)

10. J.R. Landis, G.G. Koch, The Measurement of Observer Agreement for Categorical Data, Biometrics. 33 pp. 159-74 (1977). doi:10.2307/2529310

11. S. Cafiso, B. Capace, C. D’Agostino, E. Delfino, A. Di Graziano, R. Fox-Ivey, J.. Laurent, Laser Measurement System: application on railway track, in: Tenth Int. Conf. Bear. Capacit. Roads, Railw. Airfields, (Athens, Greece, 2017)

12. S. Cafiso, A. Di Graziano, Automated in-vehicle data collection and treatment for existing roadway alignment, in: Effic. Transp. Pavement Syst. Charact. Mech. Simulation, Model. - Proc. 4th Int. Gulf Conf. Roads, pp. 785-797 (Qatar, n.d.)

13. Distress Identification manual, FHWA-RD-03-031 (FHWA, 2003)

14. ASTM D6433-11, Standard Practice for Roads and Parking Lots Pavement Condition Index Surveys, www.astm.org (ASTM International, PA, 2011)

15. SITEB, Manutenzione delle pavimentazioni stradali. Edizioni SITEBSì srl, (2004)

16. S. Cafiso, C. D’Agostino, E. Delfino, A. Montella, From manual to automatic pavement distress detection and classification, 5th IEEE Int. Conf. Model. Technol. Intell. Transp. Syst. MT-ITS 2017 - Proc. pp. 433-438 (2017). doi:10.1109/MTITS.2017.8005711

17. C. Eastman, P. Teicholz, R. Sacks, K. Liston, BIM handbook: A Guide to Building Information Modeling for Owners, Managers, Designers, Engineers, and Contractor (Hoboken, New Jersey: John Wiley \& Sons, Inc., 2011) 